\title{
Nitroxide polymer brushes prepared by surface-initiated ARGET ATRP and their selective oxidation performances
}

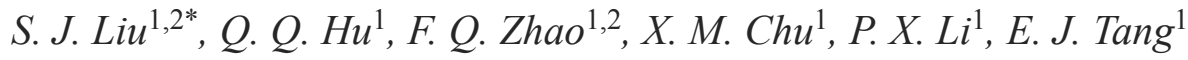 \\ ${ }^{1}$ College of Chemical \& Pharmaceutical Engineering, Hebei University of Science and Technology, 050018 Shijiazhuang, \\ PR China \\ ${ }^{2}$ Hebei Provincial Engineering and Technology Research Center of Solid Waste Utilization, 050018 Shijiazhuang, \\ PR China
}

\begin{abstract}
Polymer brushes with 2,2,6,6-tetramethyl-4-piperidyl methacrylate (TMPM) units, grafted on the cross-linked polystyrene (PS) microspheres, were synthesized via surface-initiated ARGET (activators regenerated by electron transfer) ATRP (atom transfer radical polymerization). They were further oxidized to yield nitroxide polymer brushes containing nitroxide radical units (TEMPO). The obtained polymer brushes were characterized by Fourier transform infrared spectroscopy (FT-IR), scanning electron microscopy (SEM), transmission electron microscopy (TEM), electron spin resonance (ESR) and gel permeation chromatography (GPC). The catalytic properties of nitroxide polymer brushes for selective oxidation of benzyl alcohol were investigated. The results showed that the performances were good and the yield was up to $96 \%$. Furthermore, the block brush had similar catalyst properties to non-supported TEMPO in terms of activity and selectivity. It could be recovered by centrifugation. The unity of high catalyst property and easy recovery was achieved.
\end{abstract}

Keywords: tailor-made polymers, nitroxide polymer brushes, selective oxidation, ARGET ATRP

\section{Introduction}

$\operatorname{Poly}(2,2,6,6$-tetramethylpiperidin-1-oxyl-4-yl methacrylate) (PTMA) is a kind of promising cathode-active materials for organic radical batteries [1-5]. It is a polymer containing methacrylate backbone grafted with 2, 2, 6, 6-tetramethylpiperidin-1oxyl-4-yl (TEMPO). TEMPO is a stable nitroxide radical. In order to prevent the nitroxide radical polymer dissolving into the electrolytes, Lee and coworkers [3-5] synthesized nitroxide polymer brushes which the PTMA was grafted onto the surface of silica, indium tin oxide and other substrates via surface-initiated ATRP, and the electrochemical properties were improved.

Besides, TEMPO is a good catalyst for catalytic selective oxidation of alcohol. However, the price of TEMPO is high, and its residue in the final prod- ucts may affect the product purity. In the laboratory, product can be purified by column chromatography. However, the implementation is difficult in largescale industrial production. So measures should be taken to make TEMPO recycled. To solve this problem, TEMPO has been immobilized onto inorganic (such as silica, activated carbon, molecular sieve and aluminum oxide) and organic supports (such as polymers and ionic liquids) affording solid catalysts, which are readily separated from the reaction mixtures. Using polymer as carrier, the grafting of TEMPO was easy, and some supported TEMPO showed new catalytic features compared with the non-supported TEMPO [6-16]. However, these polymer supports had, in general, a detrimental effect on the rates of reaction when compared to unsupported species. Furthermore, the supported amounts and

\footnotetext{
*Corresponding author, e-mail: sjliu16@163.com

(C) BME-PT
} 
location were also difficult to control. While the PTMA brush with TEMPO groups, it can be seen as a polymer brush supported TEMPO catalyst system. With the help of special designed brush structure and preparation method, the above problems may be solved.

Compared with the traditional ATRP method, ARGET ATRP uses significantly reduced copper catalyst concentration (down to ppm levels) and also imparts a degree of oxygen tolerance to the reaction [17]. These advantages make it a potentially more industrially attractive technique. ARGET ATRP has proven to be a very efficient tool in the preparation of polymer brushes. The used substrates include cellulose [18], polymer microspheres [19], silica [20], carbon nanotubes [21], etc. The used monomers include methyl methacrylate (MMA), styrene (St), methyl glycidyl ester of acrylic (GMA), etc. Recently, synthesis of tert-butyl methacrylate/2(dimethylamino ethyl) methacrylate based densely grafted brushes by ATRP/AGET ATRP was reported by Gromadzki et al. [22]. Stimuli-responsive polyampholyte brushes were obtained by quantitative hydrolysis of tert-butyl methacrylate units. To the best of our knowledge, grafting nitroxide polymer brushes on cross-linked PS microspheres via ARGET ATRP and using them as catalysts for selective oxidation of alcohols have not yet been reported.

In this paper, polymer brushes with TMPM units on the cross-linked PS microspheres, were synthesized via surface-initiated ARGET ATRP. Then piperidyl was oxidized by m-chloroperoxybenzoic acid (mCPBA) into nitroxide radical. The nitroxide polymer brushes were produced. These nitroxide polymer brushes were used as catalysts for selective oxidation of benzyl alcohol. With the insolubility of cross-linked PS microsphere matrix and the solubility of linear nitroxide polymer brushes, the high catalytic property and easy recovery performance were achieved.

\section{Materials and methods}

\subsection{Materials}

$\alpha$-Bromoisobutyryl bromide (98\%), copper (II) bromide $\left(\mathrm{CuBr}_{2}, 99 \%\right)$, Tin(II) 2-ethylhexanoate $\left(\mathrm{Sn}(\mathrm{EH})_{2}, 95 \%\right)$ and $\mathrm{N}, \mathrm{N}, \mathrm{N}^{\prime}, \mathrm{N}^{\prime \prime}, \mathrm{N}^{\prime \prime}$-pentamethyldiethylenetriamine (PMDETA, 99\%), were obtained from Aladdin Industrial Corporation (Shanghai, China) and used as received. m-Chloroperoxybenzoic acid (mCPBA, 75\%) purchased from J\&K
Chemical (Beijing, China) was recrystallized in methanol before use. 2,2,6,6-Tetramethyl-4-piperidyl methacrylate (TMPM, 98\%) were purchased from Tokyo Chemical Industry Co., Ltd. (Tokyo, Japan). Other monomers including styrene (St, 99\%), divinylbenzene (DVB, 55\%), hydroxyethyl methacrylate (HEMA, 96\%) and methyl methacrylate (MMA, 99\%) were offered by Aladdin Industrial Corporation (Shanghai, China). These monomers were passed through a column of neutral aluminum oxide prior to use to remove the inhibitor. Other reagents were from Sinopharm Chemical Reagent Company (China) and used without further purification.

\subsection{Synthesis of nitroxide polymer brushes on cross-linked PS microspheres}

In this study, we prepared two nitroxide polymer brushes: the homopolymer brush of PTMA and the block polymer brush of P(MMA-b-TMA). The preparation process of $\mathrm{P}(\mathrm{MMA}-\mathrm{b}-\mathrm{TMA})$ brush was illustrated in Figure 1.

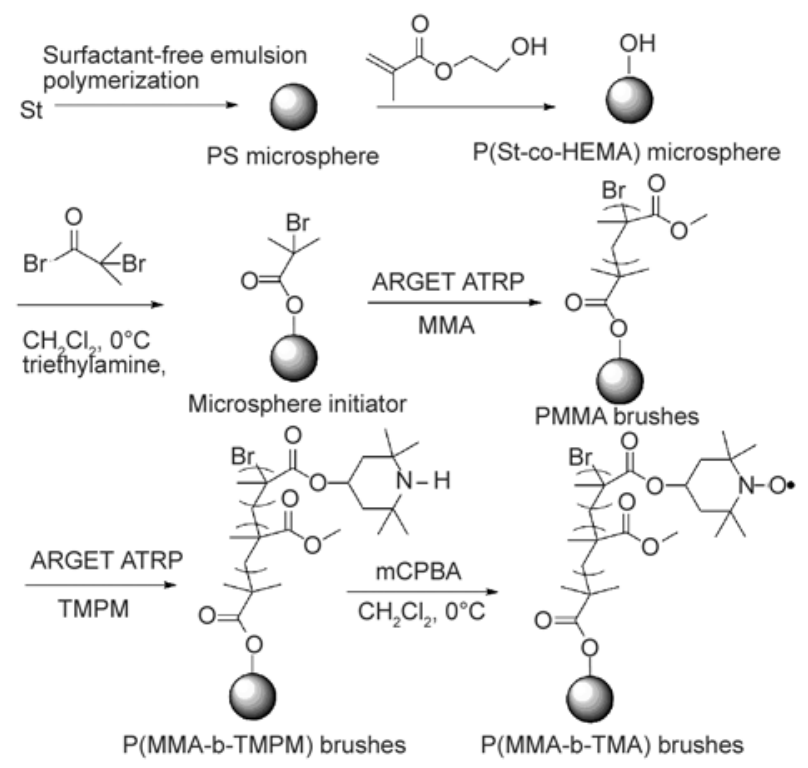

Figure 1. Synthesis of P(MMA-b-TMA) brushes on PS microspheres

\subsubsection{Preparation of cross-linked P(St-co-HEMA) microspheres by soap-free emulsion polymerization}

The cross-linked P(St-co-HEMA) microspheres were prepared by a soap-free emulsion polymerization in a $1000 \mathrm{~mL}$ round-bottom flask equipped with a mechanical stirrer and reflux condenser at $\mathrm{N}_{2}$ atmosphere. $27 \mathrm{~g} \mathrm{St}, 1.3 \mathrm{~g}$ DVB, $0.054 \mathrm{~g} \mathrm{NaOH}$, $0.054 \mathrm{~g} \mathrm{NaHCO}_{3}$ and $500 \mathrm{~mL}$ distilled water were 
added to the reactor. When the temperature rose to $75^{\circ} \mathrm{C}$, initiator solutions $(0.5 \mathrm{~g}$ potassium persulfate dissolved in $40 \mathrm{~mL}$ water) was added to initiate the polymerization. $2.7 \mathrm{~g}$ HEMA was added 5 hours later. After 3 hours' polymerization, the cross-linked $\mathrm{P}$ (St-co-HEMA) microspheres were obtained by centrifuging and drying.

\subsubsection{Immobilization of ATRP initiator on cross-linked P(St-co-HEMA) microspheres}

In an ice bath, $\alpha$-bromoisobutyryl bromide $(1.8 \mathrm{~g}$, $8 \mathrm{mmol}$ ) was added to a dispersion of microspheres (5 g) and triethylamine $(0.9 \mathrm{~g}, 9 \mathrm{mmol})$ in $\mathrm{CH}_{2} \mathrm{Cl}_{2}$ $(100 \mathrm{~mL})$. The reaction was quenched by the addition of methanol after 5 hours of gentle stirring at ambient temperature. The microspheres were centrifuged, washed with $\mathrm{CH}_{2} \mathrm{Cl}_{2}$, methanol and dried at ambient conditions.

\subsubsection{Synthesis of PTMPM brush via surface-initiated ARGET ATRP}

In nitrogen atmosphere, anisole (30 $\mathrm{mL})$ and TMPM ( $5 \mathrm{~g}, 22.2 \mathrm{mmol})$ were added to a $50 \mathrm{~mL}$ three-necked flask. After TMPM was dissolved, added $\mathrm{CuBr}_{2}$ $(4.5 \mathrm{mg}, 0.02 \mathrm{mmol})$ and PMDETA $(0.083 \mathrm{~mL}$, $0.4 \mathrm{mmol})$ at $40^{\circ} \mathrm{C}$. Then the macromolecular initiator $(1.25 \mathrm{~g}$, containing $0.4 \mathrm{mmol} \mathrm{Br}$ ) was added. When the system completely decentralized and the temperature rose to $80^{\circ} \mathrm{C}, \mathrm{Sn}(\mathrm{EH})_{2}(0.13 \mathrm{~mL}$, $0.4 \mathrm{mmol}$ ) was added to initiate the reaction. After $105 \mathrm{~min}$, the polymerization was stopped by exposing the solution to air. The PTMPM brush was obtained by centrifugation, washing with anisole, and dried in a vacuum oven at $60^{\circ} \mathrm{C}$ to constant weight.

The synthesis of block copolymer brush of P(MMAb-TMPM) was similar to the above process. Firstly PMMA brush was prepared, and then P(MMA-bTMPM) block brush was obtained through using PMMA brush as the macromolecular initiator and TMPM as the monomer.

\subsubsection{Synthesis of nitroxide polymer brushes}

PTMPM brush $(1.654 \mathrm{~g})$ was dispersed in $60 \mathrm{~mL}$ of $\mathrm{CH}_{2} \mathrm{Cl}_{2}$. Then, $20 \mathrm{~mL} \mathrm{CH} \mathrm{Cl}_{2}$ solution of mCPBA $(1 \mathrm{~g}, 5.9 \mathrm{mmol})$ was dropped at $0^{\circ} \mathrm{C}$. After $1 \mathrm{~h}$, the mixture was poured into $120 \mathrm{~mL} \mathrm{n}$-hexane, and the orange precipitate appeared. Then the pink solid product was washed and ultrasonicated again with dimethyl formamide (DMF) three times and dried in a vacuum oven for 24 hours at $60^{\circ} \mathrm{C}$ to give PTMA brush. With similar process, the P(MMA-bTMA) brush was obtained.

\subsubsection{Basic hydrolysis of polymer brush}

$50 \mathrm{~mL}$ of a $1 \mathrm{M} \mathrm{KOH} /$ ethanol solution was added to $100 \mathrm{mg}$ of polymer brush in $200 \mathrm{~mL}$ of tetrahydrofuran (THF). The mixture was refluxed at $80^{\circ} \mathrm{C}$ for $72 \mathrm{hrs}$, followed by filtration and then redissolved into THF. The resultant polymer was precipitated by the addition of acidified methanol.

\subsubsection{Characterization of polymer brushes}

The particle size of polymer microsphere was measured by laser particle size analyzer (Zetasizer Malvern Nano S90, Malvern Instruments Ltd, United Kingdom). The Br content of macromolecular initiator was estimated by Automatic Elemental Analyzer (Chncorder-MF-3, United States). The monomer conversion of polymerization was given by gravimetry method. The morphology of polymer brush was characterized by using TEM (Jeol 100CXII, Jeol Co., Japan) and SEM (S-4800-I, HITACHI, Japan). After polymer brushes cut from the substrate surface by hydrolysis, the number molecular weight $\left(M_{\mathrm{n}}\right)$ and dispersity index $(D)$ were determined using THF $(0.8 \mathrm{~mL} / \mathrm{min})$ as the mobile phase at $45^{\circ} \mathrm{C}$ by GPC (Waters GPCV 2000 with a combined refractive index and viscosity detector, United States). A universal calibration method was performed using series of narrow linear polystyrene standards. Conversion of PTMA brush from PTMPM brush was estimated by electron spin resonance (ESR, Bruker EMX-10, United States) [3].

\subsection{General process for benzyl alcohol oxidation}

$8.6 \mathrm{~mL}$ of $\mathrm{NaClO}$ aqueous solution $\left(0.121 \mathrm{~g} \cdot \mathrm{mL}^{-1}\right)$ and $8.6 \mathrm{~mL}$ of $\mathrm{NaHCO}_{3}$ aqueous solution $\left(0.05 \mathrm{~g} \cdot \mathrm{mL}^{-1}\right)$ were added to a solution of benzyl alcohol $(1.2 \mathrm{~mL}, 11.5 \mathrm{mmol})$ in $10 \mathrm{~g}$ of $\mathrm{CH}_{2} \mathrm{Cl}_{2}$ containing $1 \%$ mol of polymer brush supported TEMPO [PTMA $0.091 \mathrm{~g}$ or P(MMA-b-TMA) $0.065 \mathrm{~g}$ ] and $10 \% \mathrm{~mol}$ of $\mathrm{KBr}(0.137 \mathrm{~g}, 1.15 \mathrm{mmol})$. The reaction mixture was stirred at $0^{\circ} \mathrm{C}$ for 3 mins. Then, $\mathrm{Na}_{2} \mathrm{~S}_{2} \mathrm{O}_{3}$ was added to stop the reaction. The organic phase was separated, dried over $\mathrm{MgSO}_{4}$, and finally analyzed by gas chromatography (GC). The process was illustrated in Figure 2. 
<smiles>OCc1ccccc1</smiles>
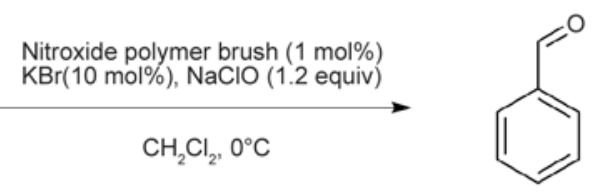

Figure 2. Oxidation of benzyl alcohol by the nitroxide polymer brush

\subsection{General procedure for the recovery of P(MMA-b-TMA) brush}

The P(MMA-b-TMA) brush was retrieved and circulated use. The brushes in aqueous phase were deposited by filtration, washing with DMF and drying. Repeated the above operation (see section 2.3) 5 times to study the catalytic properties of recycled brushes.

\section{Results and discussion}

\subsection{Synthesis of cross-linked PS microspheres with ATRP initiator}

The cross-linked surface-hydroxylated PS microspheres, P(St-co-HEMA), were prepared by soapfree emulsion polymerization. From the SEM image in Figure 3a, it was seen that P(St-co-HEMA) had the particle size of about $400 \mathrm{~nm}$, in accordance with the dynamic light scattering result (425 nm). Through the O-acylation reaction of the surface hydroxyl groups of P(St-co-HEMA) with $\alpha$-bromoisobutyryl bromide, the ATRP initiator was immobilized. The content of $\mathrm{Br}$ in these particles was about $0.32 \mathrm{mmol} / \mathrm{g}$, which was estimated by the elemental analysis result. These microspheres were used as macromolecular initiators to prepare polymer brushes.

\subsection{Synthesis of nitroxide polymer brushes}

\subsubsection{Homopolymer brush of PTMPM}

The PTMPM brush was prepared by surface-initiated ARGET ATRP. The SEM image (Figure 3b) showed that PTMPM brushes/PS nanoparticles had a slight adhesion due to the presence of polymer brushes. Similar phenomenon was reported by Lin et al. [3] when they grafted nitroxide polymer brushes on silica nanoparticles. Its FT-IR spectrum (ATR, $\mathrm{cm}^{-1}$ ) could be seen in Figure 4: $972(\mathrm{~m}), 1152$ (vs, vas $(\mathrm{OCC}=\mathrm{O})), 1236(\mathrm{~s}$, vas $(\mathrm{CC}=\mathrm{O})), 1378(\mathrm{~m}, \delta \mathrm{s}$ $\left.\left(\mathrm{CH}_{3}\right)\right), 1452\left(\mathrm{~m}, \mathrm{~d}\left(\mathrm{CH}_{2}\right)\right), 1724(\mathrm{vs}, \mathrm{v}(\mathrm{C}=\mathrm{O}))$, 2960 (m, v(CH)), 3332 (m), 3423 (m, v (NH)).

TEM images (Figure 5a, 5b) indicated that the brushes showed a core-shell structure. The core was PS matrix with a diameter of about $400 \mathrm{~nm}$ and the shell was a layer of TMPM polymer chains. Particle size of PTMPM homopolymer brush was about $500 \mathrm{~nm}$. The adhesion among the particles may be caused by the intertwining of brushes. Therefore, the PTMPM brush was successfully prepared.

Grafting density (chains $\mathrm{nm}^{-2}$ ) of polymer brush was calculated according to the Equation (1):

grafting density $=\frac{W}{M \cdot S} \cdot N_{\mathrm{A}} \cdot 10^{-18}$

where $W$ was the grafting amount of polymer brush [g] polymer brush/g matrix; $M$ was the number

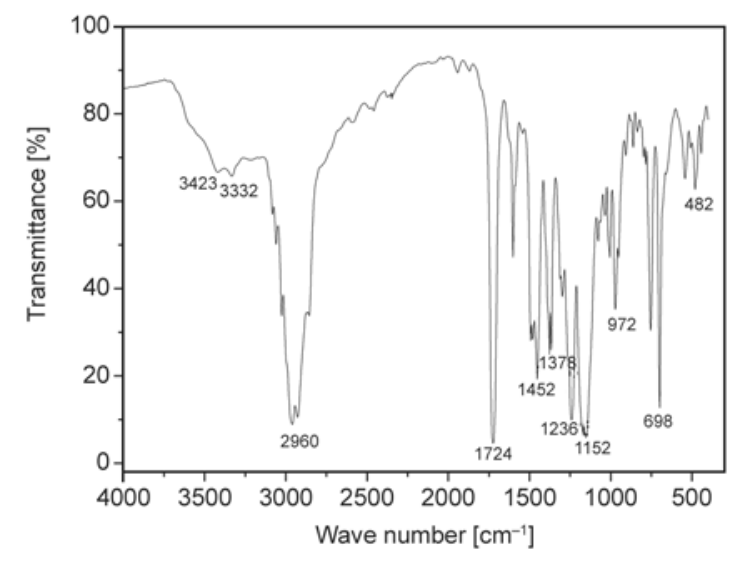

Figure 4. FT-IR spectrum for PTMPM brushes/P(St-coHEMA) microspheres

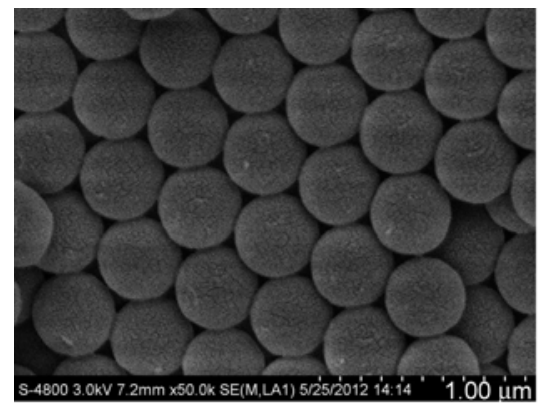

a)

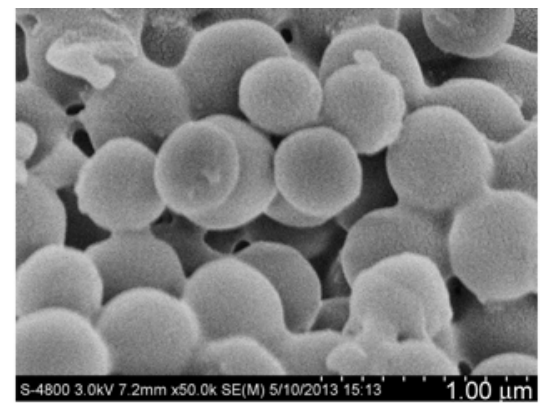

b)

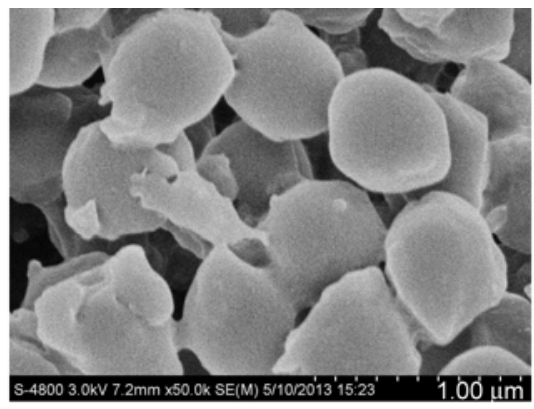

Figure 3. SEM micrographs for the bare P(St-co-HEMA) microspheres (a), PTMPM brushes/P(St-co-HEMA) microspheres (b) and P(MMA-b-TMPM) brushes/P(St-co-HEMA) microspheres (c) 


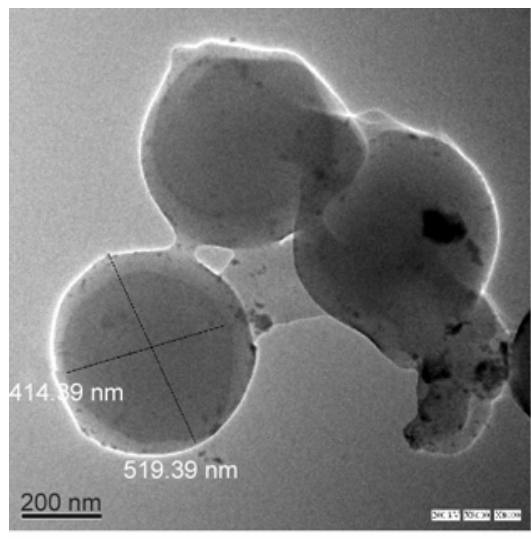

a)

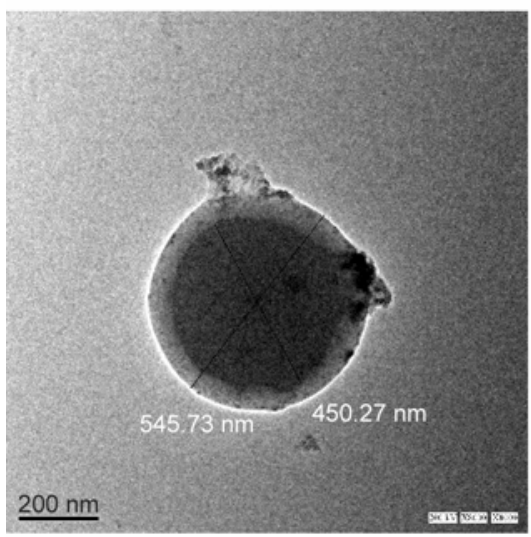

c)

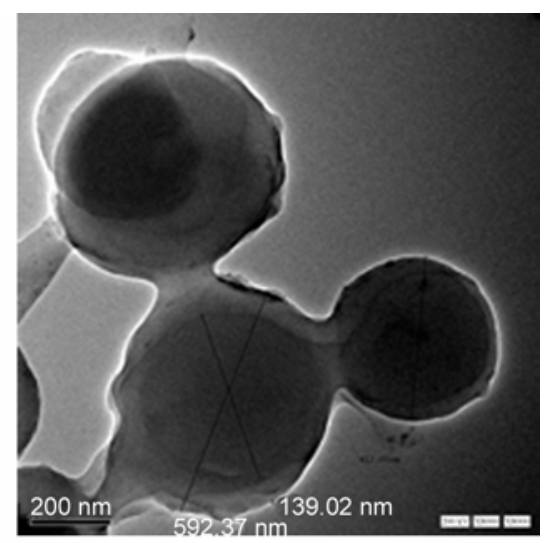

b)

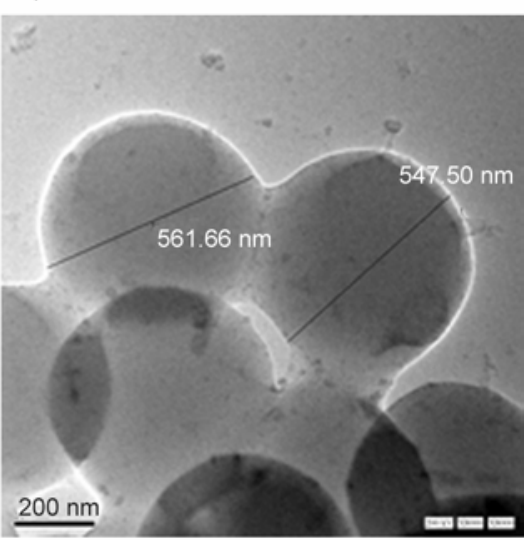

d)

Figure 5. TEM micrographs of the prepared polymer brushes: PTMPM brushes/P(St-co-HEMA) microspheres, (a) and (b); P(MMA-b-TMPM) brushes/P(St-co-HEMA) microspheres, (c) and (d)

average molecular weight of the polymer brush $[\mathrm{g} / \mathrm{mol}] . S$ was the specific surface area $\left[\mathrm{m}^{2} \cdot \mathrm{g}^{-1}\right] \cdot N_{\mathrm{A}}$ was Avogadro's number.

For the PTMPM brush, the grafting amount was $0.75 \mathrm{~g}$ TMPM brush/g substrate. Brushes were cut down from PS matrix by hydrolysis. It was measured that $M_{\mathrm{n}}=16000 \mathrm{~g} / \mathrm{mol}$ and dispersity index $(D)=$ 1.25. The grafting density was about 3.3 chains $\mathrm{nm}^{-2}$. This grafting density was higher than that on silica nanoparticles, which was about 1 chains $\mathrm{nm}^{-2}$ [3].

\subsubsection{Block copolymer brush of P(MMA-b-TMPM)}

The block copolymer brush of P(MMA-b-TMPM) was synthesized with the process as shown in Figure 1. From the SEM micrograph in Figure 3c, particles with P(MMA-b-TMPM) brushes had an irregular shape and were non-spherical. The long brushes may be intertwined with each other. It was seen from the TEM images (Figure 5c, 5d) that, the particles with block brushes also showed a coreshell structure.

The grafting amount of block copolymer brush of P(MMA-b-TMPM) was $0.69 \mathrm{~g}$ TMPM brush/g sub- strate. Its $M_{\mathrm{n}}$ was $22000 \mathrm{~g} / \mathrm{mol}$ and $D$ was 1.41 . The grafting density was about 3.8 chains $\mathrm{nm}^{-2}$.

\subsubsection{Preparation of nitroxide polymer brushes}

The piperidyl groups of PTMPM and P(MMA-bTMPM) brushes were oxidized by mCPBA into nitroxide radical, and two nitroxide polymer brushes (PTMA and P(MMA-b-TMA)) were obtained.

Because FT-IR spectra of PTMA was similar with PTMPM (the absorption peak of $\mathrm{N}-\mathrm{O}$ - was very weak) [3], so the spectrum could not be used to judge whether the reaction occurred. But during the course of the experiment, the phenomenon was obvious that the system changed from white to orange. The commercial TEMPO was orange. Furthermore, the conversion for TEMPO of the nitroxide polymer brushes were measured by ESR [3]. The conversion was about $95 \%$ after the oxidation. The nitroxide polymer brushes prepared in this work showed a high level of TEMPO immobilization, $3.1 \mathrm{mmol} \cdot \mathrm{g}^{-1}$ for PTMA and $3.6 \mathrm{mmol} \cdot \mathrm{g}^{-1}$ for $\mathrm{P}(\mathrm{MMA}-\mathrm{b}-\mathrm{TMA})$. This density level is considerably higher than that of the TEMPO polymer grafted on silica (up to $2.1 \mathrm{mmol} \cdot \mathrm{g}^{-1}$ ) [23]. 


\subsection{The performance of nitroxide polymer brushes for catalytic oxidation of benzyl alcohol}

With $\mathrm{NaClO}$ as the oxidant, the nitroxide polymer brushes as catalysts, benzyl alcohol was selectively oxidized to benzaldehyde. The catalyst performance of nitroxide polymer brushes was compared with non-supported TEMPO. GC results showed that the system possessed excellent selectivity using non-supported TEMPO, PTMA brush and P(MMAb-TMA) brush as catalysts. No characteristic peaks of benzoic acid were found. The yield of benzaldehyde was, 95, 91 and 96\%, respectively. Block copolymer brush of P(MMA-b-TMA) had similar results with non-supported TEMPO. It may be that the special structure of block brushes made TEMPO groups mainly distributed in the outer of particles, which was helpful to fully contact with the reactants.

Recycling performance of the P(MMA-b-TMA) brush was investigated after the filtration recovery. The yield results for 5 cycles, were shown in Figure 6. It was found that the yield did not fall significantly after 5 cycles. Furthermore, benzoic acid was not detected. The high catalytic activities and preservation of activity upon recycling of these brushes may be attributed to enhanced regeneration of the nitroxyl species as a result of intramolecular synproportionation [10]. The oxidation for other primary alcohols and seconday alcohols is under work.

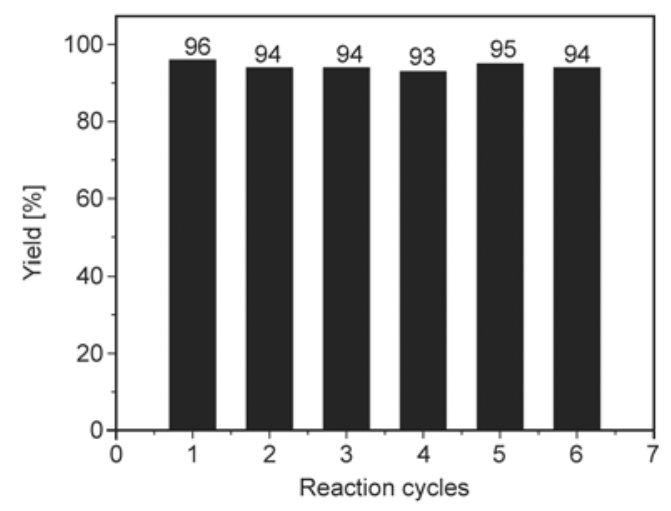

Figure 6. Recyclability of P(MMA-b-TMA) brushes in the oxidation of benzyl alcohol to benzaldehyde

\section{Conclusions}

The surface-hydroxylated cross-linked PS microspheres were prepared by soap-free emulsion poly- merization, and then ATRP initiator was supported on them. Using these microspheres as macromolecular initiators, the homopolymer brush of PTMPM and block copolymer brush of P(MMA-b-TMPM) were synthesized via surface-initiated ARGET ATRP, and the grafting density was 3.3 and 3.8 chains $\mathrm{nm}^{-2}$ respectively. PTMPM and P(MMA-b-TMPM) brushes were further oxidized by mCPBA to yield nitroxide polymer brushes of PTMA and P(MMAb-TMA) with TEMPO groups. With $\mathrm{NaClO}$ as oxidant, selective oxidation of benzyl alcohol to benzaldehyde as template reaction, the catalytic performances of nitroxide polymer brushes were studied. The results showed that the brushes had excellent catalytic properties, and the P(MMA-bTMA) brush demonstrated an equivalent catalytic performance with the non-supported TEMPO. The catalyst system was easy to recycle, and the yield did not fall significantly after 5 cycles.

\section{Acknowledgements}

This study was supported by Natural Science Foundation of China (NO. 21304030), Natural Science Foundation of Hebei Province (NO. B2012208017) and Outstanding Youth Fund in Hebei Province Department of Education (NO. YQ2013001). Here we expressed the heartfelt thanks for the support.

\section{References}

[1] Janoschka T., Teichler A., Krieg A., Hager M. D., Schubert U. S.: Polymerization of free secondary amine bearing monomers by RAFT polymerization and other controlled radical techniques. Journal of Polymer Science Part A: Polymer Chemistry, 50, 1394-1407 (2012). DOI: $10.1002 /$ pola.25907

[2] Hauffman G., Rolland J., Bourgeois J-P., Vlad A., Gohy J-F.: Synthesis of nitroxide-containing block copolymers for the formation of organic cathodes. Journal of Polymer Science Part A: Polymer Chemistry, 51, 101108 (2013).

DOI: $10.1002 /$ pola.26279

[3] Lin H-C., Li C-C., Lee J-T.: Nitroxide polymer brushes grafted onto silica nanoparticles as cathodes for organic radical batteries. Journal of Power Sources, 196, 8098-8103 (2011).

DOI: $10.1016 /$ j.jpowsour.2011.05.037

[4] Wang Y-H., Hung M-K., Lin C-H., Lin H-C., Lee J-T.: Patterned nitroxide polymer brushes for thin-film cathodes in organic radical batteries. Chemical Communications, 47, 1249-1251 (2011).

DOI: $10.1039 / \mathrm{c} 0 \mathrm{cc} 02442 \mathrm{~b}$ 
[5] Lin C-H., Chou W-J., Lee J-T.: Three-dimensionally ordered macroporous nitroxide polymer brush electrodes prepared by surface-initiated atom transfer polymerization for organic radical batteries. Macromolecular Rapid Communications, 33, 107-113 (2012). DOI: $10.1002 /$ marc. 201100548

[6] Dijksman A., Arends I. W. C. E., Sheldon R. A.: Polymer immobilised TEMPO (PIPO): An efficient catalyst for the chlorinated hydrocarbon solvent-free and bromide-free oxidation of alcohols with hypochlorite. Chemical Communications, 2000, 271-272 (2000). DOI: 10.1039/A909690F

[7] Dijksman A., Arends I. W. C. E., Sheldon R. A.: A comparison of the activity of polymer immobilised TEMPO (PIPO) with MCM-41 and silica supported TEMPO as heterogeneous catalysts for the oxidation of alcohols. Synlett, 2001, 0102-0104 (2001).

DOI: $10.1055 / \mathrm{s}-2001-9708$

[8] Ferreira P., Hayes W., Phillips E., Rippon D., Tsang S. C.: Polymer-supported nitroxyl catalysts for selective oxidation of alcohols. Green Chemistry, 6, 310-312 (2004).

DOI: $10.1039 / \mathrm{B} 406829 \mathrm{G}$

[9] Ferreira P., Phillips E., Rippon D., Tsang S. C., Hayes W.: Poly(ethylene glycol)-supported nitroxyls: Branched catalysts for the selective oxidation of alcohols. The Journal of Organic Chemistry, 69, 6851-6859 (2004). DOI: $10.1021 /$ jo0490494

[10] Ferreira P., Phillips E., Rippon D., Tsang S. C.: Catalytic oxidation of alcohols using molecular oxygen mediated by poly(ethylene glycol)-supported nitroxyl radical. Applied Catalysis B: Environmental, 61, 206211 (2005). DOI: $10.1016 /$ j.apcatb.2005.05.006

[11] Pozzi G., Cavazzini M., Quici S., Benaglia M., Dell'Anna G.: Poly(ethylene glycol)-supported TEMPO: An efficient, recoverable metal-free catalyst for the selective oxidation of alcohols. Organic Letters, 6, 441-443 (2004).

DOI: $10.1021 / 01036398 w$

[12] Benaglia M., Puglisi A., Holczknecht O., Quici S., Pozzi G.: Aerobic oxidation of alcohols to carbonyl compounds mediated by poly(ethylene glycol)-supported TEMPO radicals. Tetrahedron, 61, 12058 12064 (2005).

DOI: $10.1016 /$ j.tet.2005.07.107

[13] Gilhespy M., Lok M., Baucherel X.: Polymer-supported nitroxyl radical catalysts for the hypochlorite and aerobic oxidation of alcohols. Catalysis Today, 117, 114-119 (2006). DOI: $10.1016 /$ j.cattod.2006.05.039
[14] Gilhespy M., Lok M., Baucherel X.: Polymer-supported nitroxyl radical catalyst for selective aerobic oxidation of primary alcohols to aldehydes. Chemical Communications, 2005, 1085-1086 (2005).

DOI: $10.1039 / \mathrm{B} 415902 \mathrm{~K}$

[15] Tanyeli C., Gümüs A.: Synthesis of polymer-supported TEMPO catalysts and their application in the oxidation of various alcohols. Tetrahedron Letters, 44, 1639-1642 (2003).

DOI: $10.1016 / \mathrm{S} 0040-4039(03) 00003-0$

[16] Kashiwagi Y., Ikezoe H., Ono T.: Oxidation of alcohols with nitroxyl radical resins under two-phase conditions. Synlett, 2006, 69-72 (2006).

DOI: $10.1055 / \mathrm{s}-2005-922775$

[17] Braunecker W. A., Matyjaszewski K.: Controlled/living radical polymerization: Features, developments, and perspectives. Progress in Polymer Science, 32, 93-146 (2007).

DOI: $10.1016 /$ j.progpolymsci.2006.11.002

[18] Hansson S., Östmark E., Carlmark A., Malmström E.: ARGET ATRP for versatile grafting of cellulose using various monomers. ACS Applied Materials and Interfaces, 1, 2651-2659 (2009).

DOI: $10.1021 / \mathrm{am} 900547 \mathrm{~g}$

[19] Jonsson M., Nyström D., Nordin O., Malmström E.: Surface modification of thermally expandable microspheres by grafting poly(glycidyl methacrylate) using ARGET ATRP. European Polymer Journal, 45, 23742382 (2009).

DOI: 10.1016/j.eurpolymj.2009.05.002

[20] Cheesman B. T., Willott J. D., Webber G. B., Edmondson S., Wanless E. J.: pH-responsive brush-modified silica hybrids synthesized by surface-initiated ARGET ATRP. ACS Macro Letters, 1, 1161-1165 (2012). DOI: $10.1021 / \mathrm{mz} 3003566$

[21] Aitchison T. J., Markovic M-G., Saunders M., Fredericks P., Valiyaveettil S., Matisons J. G., Simon G. P.: Polymer brushes on multiwalled carbon nanotubes by activators regenerated by electron transfer for atom transfer radical polymerization. Journal of Polymer Science Part A: Polymer Chemistry, 49, 4283-4291 (2011). DOI: $10.1002 /$ pola.24872

[22] Gromadzki D., Štěpánek P., Makuška R.: Synthesis of densely grafted copolymers with tert-butyl methacrylate/2-(dimethylamino ethyl) methacrylate side chains as precursors for brush polyelectrolytes and polyampholytes. Materials Chemistry and Physics, 137, 709715 (2013).

DOI: $10.1016 / \mathrm{j}$. matchemphys.2012.09.012

[23] Saito K., Hirose K., Okayasu T., Nishide H., Hearn M. T. W.: TEMPO radical polymer grafted silicas as solid state catalysts for the oxidation of alcohols. RSC Advances, 3, 9752-9756 (2013).

DOI: $10.1039 / \mathrm{C} 3 \mathrm{RA} 41823 \mathrm{E}$ 